\title{
BMJ Open Restaurant interventions for salt reduction in China: protocol for a randomised controlled trial
}

\author{
Wenwen Du (D) , ${ }^{1}$ Jiguo Zhang, ${ }^{1}$ Yuan $\mathrm{Li}^{2}{ }^{2}$ Feng $\mathrm{J} \mathrm{He},{ }^{3}$ Xue Zhou, ${ }^{4}$ Zhihua Xu (D) , ${ }^{5}$ \\ Yifu Gao, ${ }^{6}$ Lei Yin, ${ }^{7}$ Xiaoyu Chang, ${ }^{8}$ Wei Yan, ${ }^{9}$ Monique Tan (D) ${ }^{3}$ \\ Graham A MacGregor, ${ }^{3}$ Rong Luo, ${ }^{2}$ Puhong Zhang, ${ }^{2}$ Huijun Wang ${ }^{1}$
}

To cite: Du W, Zhang J, Li Y, et al. Restaurant interventions for salt reduction in China: protocol for a randomised controlled trial. BMJ Open 2020;10:e038744. doi:10.1136/ bmjopen-2020-038744

- Prepublication history for this paper is available online. To view these files, please visit the journal online (http://dx.doi org/10.1136/bmjopen-2020038744).

Received 25 March 2020 Revised 18 September 2020 Accepted 08 October 2020
Check for updates

(C) Author(s) (or their employer(s)) 2020. Re-use permitted under CC BY-NC. No commercial re-use. See rights and permissions. Published by BMJ.

For numbered affiliations see end of article.

Correspondence to Professor Huijun Wang; wanghj@ninh.chinacdc.cn and Dr Puhong Zhang;

zpuhong@georgeinstitute.org.cn

\section{ABSTRACT}

Introduction Salt intake in China is high, and most of it comes from that added by consumers. Nevertheless, recent years have seen a rapid increase in the frequency at which people eat out. The aim of this study is to evaluate the effectiveness of interventions designed for salt reduction in restaurants through a randomised controlled trial in China.

Methods and analysis As a randomised controlled trial with restaurants as study subjects, we recruited 192 restaurants from 12 counties of 6 provinces in China. After the baseline survey, restaurants were randomly assigned to intervention or control group. Using social cognitive theory, comprehensive intervention activities were designed to encourage salt reduction in all restaurant foods, and at the same time, to encourage consumers to choose lower salt options when eating out. The interventions will be conducted only in restaurants of the intervention group during the first year. The followup assessment will be conducted at the end of the trial. The primary outcome is the change in the average salt content of the five best-selling dishes of the restaurant, as measured by laboratory tests. Secondary outcomes include differences in the monthly use of salt and salty condiments between intervention and control restaurants, and the knowledge, attitude and practice on salt among restaurant consumers.

Ethics and dissemination The study was reviewed and approved by the Review Board of the National Institute for Nutrition and Health, Chinese Center for Disease Control and Prevention and Queen Mary Research Ethics Committee. Results will be disseminated through presentations, publications and social media.

Trial registration number ChiCTR1800019694; Preresults.

\section{INTRODUCTION}

High salt intake is one of the leading dietary risk factors for deaths and disability globally ${ }^{1}$ and associated with 3 million deaths and 70 million Disability Adjusted Life Years (DALYs) in adults around the world. In China, high salt intake attributed to more than 0.5 million cardiometabolic deaths in 2010-2012. ${ }^{2}$ The most common risk of high salt intake is raised blood pressure (BP), which alone accounted

\section{Strengths and limitations of this study}

- The study develops an effective and sustainable intervention package for salt reduction in Chinese restaurant settings.

- Our study covers a wide range of restaurants in China, from six provinces and thus representing different cuisines and eating habits.

Due to the commercial nature of restaurants, the implementation of the salt reduction interventions may be challenging and need strong multisector support and cooperation.

for an estimated 10.7 million deaths each year worldwide. ${ }^{3}$ Statistics from the 2015 China's Report on Nutrition and Chronic Diseases revealed that the prevalence of hypertension among the Chinese population aged 18 years and older was $25.2 \%$ in $2012,{ }^{4}$ with the total number of individuals with high BP reaching 270 million. International experience had proved that reducing population salt intake lowered $\mathrm{BP}$ and reduced the risk of cardiovascular disease (CVD) ${ }^{56}$ Salt reduction is considered one of the most costeffective measures to improve public health. ${ }^{7}$ In China, the average salt intake was $12-14 \mathrm{~g}$ salt per day, which was more than double the WHO-recommended maximum level of $5 \mathrm{~g}$ salt per day for adults. ${ }^{89}$

With the rapid urbanisation and lifestyle changes in China, eating in restaurants has been becoming popular, especially in urban areas. National survey (2010-2012) statistics showed that $35.5 \%$ of the Chinese aged 6 years and older had eaten out in the past week. The proportion of people dining out was $42.2 \%$ and $28.5 \%$ in urban and rural areas, respectively. ${ }^{4}$ Restaurants became the second major dining location after home cooking in China, and this had important impacts on public health and nutrition. A pilot study using a 1-week salt estimation 
method in Beijing found that approximately $40 \%$ of the salt intake was consumed outside the home. ${ }^{10}$ Restaurant dishes seemed to have a high sodium content in both developed and developing countries. ${ }^{11-14}$ A study showed that $46.8 \%$ of the dishes served in Chinese restaurants in Beijing contained more salt than the daily salt intake recommended by WHO. ${ }^{14}$ Sodium content was substantially higher in restaurant foods than in home-made foods. ${ }^{15-17}$ Therefore, to help consumers reduce their overall salt intake, it is important to develop an effective strategy to reduce the sodium content of restaurant foods.

To tackle the high salt intake levels in China, Action on Salt China (ASC) was set up in 2017, funded by the UK National Institute for Health Research. ASC aimed to implement comprehensive national salt reduction programmes, with the leadership of Queen Mary University of London, the George Institute China, Chinese Center for Disease Control and Prevention (China CDC) and other key related organisations. ${ }^{18}$ The ASC team developed two national health campaigns (health education and salt reduction in packaged food) and four randomised controlled trials (RCTs) to test interventions targeting the major sources of salt intake. ${ }^{19}$ The RCTs consisted of: (1) an application-based intervention study in schoolchildren and their families ${ }^{20}$; (2) a home cookbased intervention study; (3) a comprehensive intervention study ${ }^{21}$; and (4) a restaurant-based intervention study (RIS), which is the one reported in the present paper.

The objective of RIS was to reduce salt use by at least $0.5 \mathrm{~g}$ per $100 \mathrm{~g}$ in restaurant dishes. To achieve the goal, we developed a restaurant salt reduction package, the feasibility and effectiveness of which are being tested by RIS. The key interventions were based on social cognitive theory $^{22}$ and included: (1) building tailored restaurant environments that encourage consumers to order lowersalt or reduced-salt dishes; (2) lower salt or reduced-salt ordering reminders from the waiters; (3) training cooks in reduced-salt cooking; and (4) salt reduction campaigns. As a part of the ASC programme, this article reports on the design of the RIS intervention package and its implementation, evaluation and current status.

\section{METHODS AND ANALYSIS}

\section{Study setting and sampling method}

To account for geographical, economic and dietary disparities, our study was carried out in six provinces of China, which were consistent with other RCTs of ASC, covering north (Heilongjiang, Hebei and Qinghai) and south (Hunan, Sichuan and Jiangxi) China. As a RCT, the RIS baseline survey, which included the assessment of laboratory sodium level of five best-selling dishes, restaurant environment and attitude on salt reduction, detailed recipe information and consumers' knowledge, attitude and practice (KAP) on salt reduction in each restaurant, was conducted in May 2019, and an evaluation survey with the same assessments will be conducted after 1-year follow-up, at the end of the trial. One hundred and ninety-two restaurants were selected from 12 counties of the above-mentioned six provinces. In each province, two counties of similar socioeconomic level in the provincial capital city were selected. The counties that had participated in other salt-reduction projects were excluded to minimise contamination. To determine the effectiveness of the salt reduction package in restaurants of different sizes, of the 16 Chinese restaurants selected from each county, it was ensured these included four large restaurants, eight medium restaurants and four small restaurants. The restaurants in each county were then randomly allocated to the intervention or control group after the baseline survey, using the random procedure on a customized electronic data capture system (EDC). The comprehensive intervention package was designed to both inspire the consumers to demand for lower salt dishes and promote the skills of reduced-salt cooking and ordering service in restaurant staff.

\section{Restaurant inclusion criteria and recruitment}

First, we released an open letter via local media and cooperated with the Market Regulation Bureau to recruit restaurants that were interested in participating in the programme. Then the standard inclusion criteria were used to screen for potential restaurants, including: (1) restaurants mainly offering Chinese cuisine; (2) agreeing to participate in 1 year of comprehensive salt reduction intervention and in at least two assessment surveys (baseline and follow-up surveys); (3) with complete records of salt and other condiments purchase and usage; (4) had been operating normally for more than 1 year and without plan of relocating or closing in the next 2 years; and (5) with $>50 \%$ of the dishes that could be prepared with less salt. Restaurants that were already involved in other salt reduction programmes will be excluded. Restaurant size was determined based on surface area or number of seats (table 1). Finally, 16 restaurants in each country were selected according to the above inclusion and exclusion criteria, including four large-size, eight medium-size and four small-size restaurants.

\section{Randomisation}

After baseline assessment, restaurants were randomly allocated to either the control group or the intervention group (96 restaurants in each group). The randomisation was stratified by the size of restaurants and carried out using computer generated random numbers by a statistician who was not involved in the study and blind to the identity of the restaurants. Following the baseline survey,

\begin{tabular}{lll}
\hline Table 1 Restaurant size classification \\
\hline Classification & $\begin{array}{l}\text { Square metres } \\
\left(\mathbf{m}^{2}\right)\end{array}$ & $\begin{array}{l}\text { (or) Number of } \\
\text { seats }\end{array}$ \\
\hline Large restaurant & $>500$ and $\leq 3000$ & $>250$ and $\leq 1000$ \\
Medium restaurant & $>150$ and $\leq 500$ & $>75$ and $\leq 250$ \\
Small restaurant & $\leq 150$ & $\leq 75$ \\
\hline
\end{tabular}




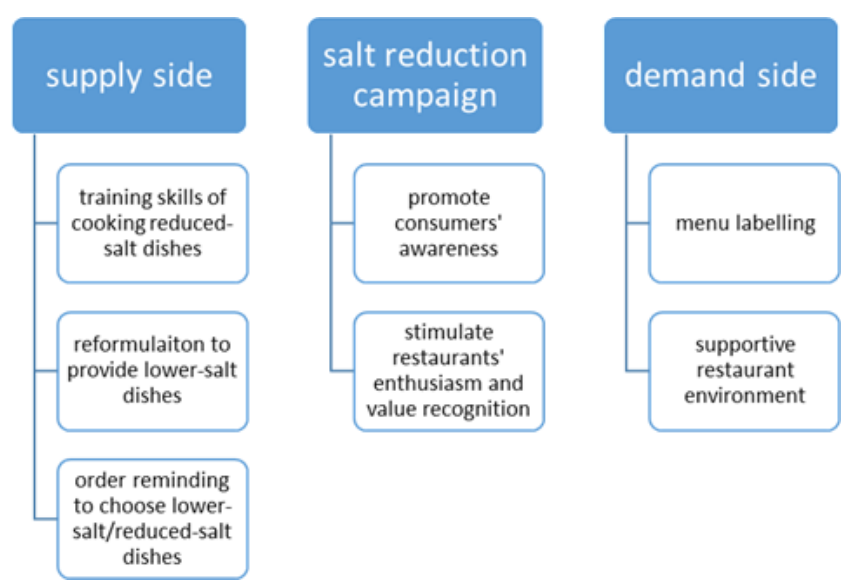

Figure 1 The RIS intervention design. RIS, restaurant-based intervention study.

the restaurants allocated to the intervention group implemented a series of salt reduction activities. Meanwhile, the control restaurants operated as usual.

\section{Intervention}

The objective of RIS was to reduce salt use in restaurants. We aimed to achieve short term and sustainable longterm effects. Social cognitive theory proposes behaviour was influenced by the constant interaction of personal factors (ie, skills and knowledge) and environmental factors (ie, appropriate modelling for learning and available materials). ${ }^{22}$ Therefore, we designed the intervention activities based on both the supply (restaurant) and demand (consumer) sides (figure 1). For the intervention group, the RIS intervention package included the following activities:

\section{Menu labelling}

In the baseline survey, we collected the detailed ingredients composition, including the amount of salt and condiments, of the 50 best-selling dishes of each restaurant. If a restaurant offers fewer than 50 dishes, information on all available dishes were collected. In each restaurant, the $10 \%$ of the dishes that had the lowest sodium content (according to baseline survey) were labelled 'lower salt' on the menu, thus providing clear information to the customers. This menu will be used in the intervention restaurants throughout the 1-year trial period.

\section{Training for chef and waiter/waitress}

In close collaboration with culinary experts, we developed a series of training materials, including manual and videos, to guide chefs and waiters/waitresses in encouraging salt reduction services in their routine work. Besides, we organised the service of a professional team to offer face-to-face training for the restaurant staff of each county at least once a year. At least three representatives per restaurant were required to attend the training. This training mainly focused on the following aspects: 'salt sources in restaurant dishes', 'why reduce salt', 'practical skills in reducing salt for restaurant cooking', 'building a reduced-salt environment in a restaurant' and 'service and communication skills'. Local county investigators were responsible to enhance the knowledge and skills among staff in each intervention restaurant by conducting monthly follow-up supervisions.

During the trial, each intervention restaurant was encouraged to reduce salt usage by $10 \%$ in all dishes and greater reductions according to the customers' request (such as $-30 \%$ or $-50 \%$ salt). Furthermore, at least three lower salt dishes (sodium $\leq 100 \mathrm{mg} / 100 \mathrm{~g}$ ) per restaurant should be developed through reformulation to provide customers more options of lower salt dishes. Waiters/ waitresses were required to recommend customers the lower salt dishes, as well as remind them they can choose the reduced-salt option with almost all the other dishes.

\section{Supportive environment for salt reduction in restaurants}

Information that refers to salt reduction, salt and health, and available reduced-salt dishes were shown through videos, posters, brochures, leaflets and table displays to build restaurant environments that make it easier for the customers to choose lower salt options. For example, messages with announcement ' $-30 \% /-50 \%$ salt options were available for most dishes' will be posted on dining tables. These materials should be displayed at noticeable positions in the intervention restaurants during the trial period.

\section{Salt reduction campaign}

To create a social environment supportive of salt reduction, local investigators were encouraged to organise a campaign at least once during the 1-year trial, with the theme of 'less salt, healthy eating', to help raise consumers' awareness of salt reduction when they eat out. Another import aim was to encourage restaurants to pay more attention to reducing salt and offering lower salt dishes. To expand the reach of the campaign, news agencies and social media were used to disseminate the campaign messages. To limit contamination to control group, the salt reduction campaign should only involve the restaurants in intervention group.

Certified medals of 'ASC Salt Reducing Restaurant' were granted to intervention restaurants, according to the standardised requirements. The medal will help motivate restaurants for salt reduction and value their efforts.

\section{Sample size}

In the current randomised controlled trial, selected restaurants were the study subjects. Based on the results of a study on Chinese restaurants, ${ }^{23}$ we assumed an SD of 1 $\mathrm{g} / 100 \mathrm{~g}$ of dish for the sodium content of Chinese restaurant dishes and expected that a sample of 192 restaurants would achieve $80 \%$ power (with two-sided alpha $=0.05$ ) to detect a change in salt content by $0.5 \mathrm{~g} / 100 \mathrm{~g}$ dish for the five best-selling dishes in each restaurant, allowing for a $20 \%$ dropping rate of restaurants. Therefore, a total of 192 restaurants were recruited into the study. 


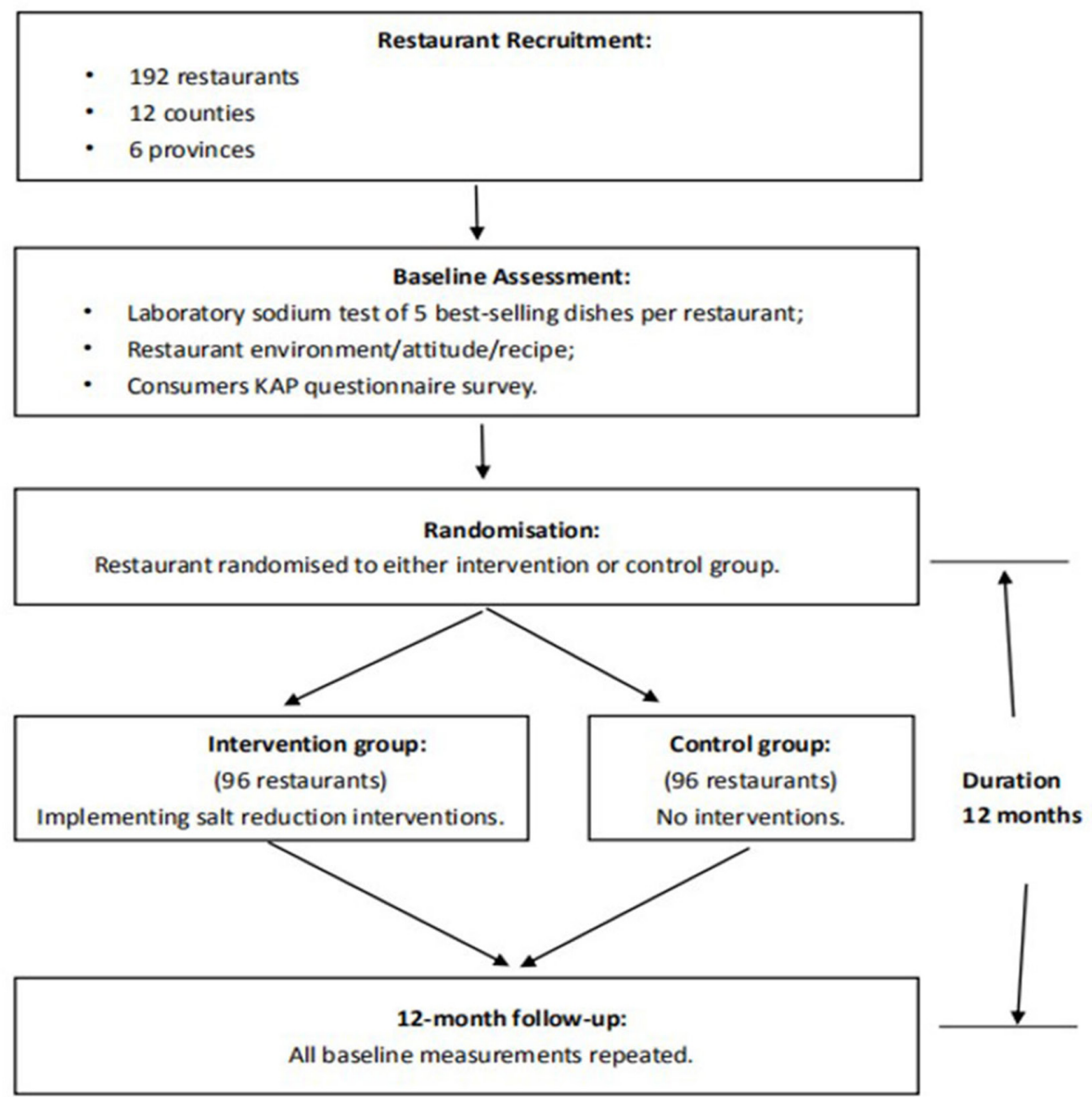

Figure 2 RIS trial design. KAP, knowledge, attitude and practice; RIS, restaurant-based intervention study.

\section{Outcome measures}

\section{Effectiveness outcomes}

The primary outcome was the differences between the intervention and control groups in the change of the sodium content of the five best-selling dishes from baseline to the end of the trial (figure 2).

Secondary outcomes included the differences between the intervention and control groups in: (1) monthly use of salt and main salty condiments by the restaurant chefs and (2) salt-related KAP in restaurant customers.

\section{Feasibility outcomes}

The feasibility outcomes included two dimensions: (1) the cost-benefit values, and (2) the fidelity and adoption of the interventions (frequency, coverage and satisfaction), as well as the barriers and facilitators of conducting the interventions.

\section{Outcome assessments}

\section{Effectiveness outcome assessments}

The primary and secondary effectiveness outcomes should be assessed before and after the 1-year intervention, in both intervention and control restaurants.

For the primary outcome, we collected the whole portion of the five best-selling dishes of each restaurant at baseline and follow-up to test their sodium content using laboratory flame atomic absorption spectrometry method. Although it would be possible to estimate the sodium content of a dish by asking the chefs what ingredients and condiments they have used, this would rely on the chefs' memory. By using laboratory tests, we ensured the accurate assessment of the sodium content of the dishes. The dishes chosen for laboratory test should be top-selling first, and then involve different types according to the ingredients (ie, animal food based dishes, mixed food dishes and vegetable food based dishes). The local researchers went to each restaurant and bought the five best-selling dishes anonymously in order to avoid introducing bias. The whole dishes, including sauce and soup when appropriate, were weighed and photographed. Once cooled off, they were transferred to a sampling bag. The samples were stored at $-20^{\circ} \mathrm{C}$ freezers until transferred to the laboratory. Theoretically, for each restaurant, the same dishes bought at a similar date of the year should be assessed at baseline and follow-up assessment. However, if the dishes collected at baseline are no longer sold at the follow-up assessment, comparable dishes with similar ingredients and cooking method could be chosen as alternatives.

For the secondary outcomes, questionnaires were administered by trained local investigators through a mobile EDC app developed for RIS. Compared with traditional data collection methods, the mobile EDC 
had advantages in terms of process and quality control, as demonstrated in a previous clinical trial. ${ }^{24}$ There were two questionnaires in the survey, including restaurant assessment and consumer survey. The questions related to the restaurant assessment consisted of: (1) basic information about the restaurant; (2) salt reduction environment and services in restaurant; (3) monthly salt and salty condiments usage; (4) attitude and challenges related to reducing salt in restaurant; and (5) dishes ( $\leq 50$ dishes/ restaurant) recipe (all ingredients and condiments used and in what quantity) and cooking method. The restaurant-related questions were asked to the owners/ managers who directly manage the restaurant, and the recipes were collected by face-to-face interview with chefs who were familiar with preparing those dishes. To assist chefs in remembering accurately the amount of ingredients used in the dishes, the investigators could show them the weighed amount using a usual spoon or other measuring instruments used in their restaurants.

For the consumer survey, we randomly selected 20 customers (10 males and 10 females) in all intervention and control restaurants, before and after the trial, to assess the changes and differences of knowledge, attitudes and behaviours related to salt reduction.

\section{Feasibility outcome assessments}

Economic evaluation will be conducted from the health sector perspective to compare the comprehensive intervention package for restaurant on salt reduction versus business as usual, and it will include two dimensions: a trial-based economic evaluation and a modelled economic evaluation of long-term costs and outcomes. Intervention costs will include the direct costs of running the programme, excluding any research and development costs. We will consider restaurant dishes consumption at the population level for the economic evaluation. Therefore, the trial-based economic evaluation will be assessed in terms of incremental cost per unit salt reduction in restaurant dishes and systolic BP. The conversion of sodium in restaurant dishes to daily sodium intake for the population, and its relationship with systolic BP, will be based on the scientific literature and other population trials under the ASC project. The modelled economic evaluation will examine the cost, survival and health states (including death and CVD events) to estimate incremental cost per life year saved and cost per quality-adjusted life year gained. The transition probabilities across health states and costs attached to different health states, and the long-term effects of reduction in sodium intake will be based on literature data. Sensitivity analyses will be used to estimate uncertainty about the primary findings associated with various key parameters. The process evaluation will help us assess the fidelity and adoption of key components of intervention (frequency, coverage and satisfaction) and understand the barriers and facilitators of the intervention. The evaluation will be conducted using mixed-methods during and at the end of the trial, from monthly supervision records, structured process evaluation form and in-depth interviews with restaurant staff and customers. Designed forms, including sales form of lower salt labelling dishes, recipe information form of reduced-salt dishes and consumer feedback cards will be collected at three time points during the intervention period, in order to monitor the acceptance and the effect of the RIS intervention.

\section{Data collection, management and analysis Data collection}

We used the specially designed mobile EDC to collect assessments data during the RIS programme, as well as monthly supervision records in the 1-year comprehensive intervention. The local CDCs were responsible for data collection. The structure of data collection system consisted of 192 restaurants. The local researchers logged in the system, input the assessment data and supervision records in each restaurant page. The assessment data included: (1) information on restaurants' salt reduction-related environment, service, attitude, challenges and monthly salt usage, as well as some sales data; (2) information on recipe, cooking method and laboratory sodium content; and (3) information on customer knowledge, attitude and behaviours related to salt reduction. To collect the primary indicator laboratorymeasured sodium contents of the best-selling dishes, the local investigators went to each restaurant and buy the five best-selling dishes anonymously, then put them totally into the sampling bags, weighed the amount, stored at $-20^{\circ} \mathrm{C}$ in refrigerator and finally transferred to the designated laboratory for sodium test. Monthly supervision records included information on the process of intervention activities, and any reason for not carrying them out, as well as photos that could inform on the intervention status.

\section{Data management}

The mobile EDC used in the RIS programme was developed by the Beijing University of Aeronautics and Astronautics. The security of data management was demonstrated in another publication under the same ASC project. ${ }^{20}$ To ensure the data validation and detection of keying errors, the EDC system set rules of logic jump for associated questions and abnormal values recognition. To guarantee the integrity and authenticity of data collection, local researchers were given different level of authority according to their roles. For example, the local investigators were responsible for inputting survey and supervision data but could not delete the records they added. The inspectors had authority to check the data accuracy, delete mistaken records, start and close discussion about doubtful data but cannot add new records. The person in charge at county, provincial and national levels could view the data of all restaurants under their management but could not make any changes. All modifications were clearly recorded in the EDC system. 


\section{Statistical analysis}

The effect of the intervention package for restaurants on the primary or secondary outcomes will be determined using linear mixed models. The differential change by groups from baseline to the end of follow-up will be indicated by including group (intervention and control), time (baseline and follow-up) and the interaction of group*time, with adjustments for potential confounding variables (area, restaurant size, cooking method and dish category). We will consider sensitivity analyses to examine the robustness of the conclusion of the primary analysis. Results will be described as mean, SD, SE and 95\% CI where appropriate. SAS V.9.4 will be used for the data cleaning and statistical analyses. All analyses will be two sided, and $\mathrm{p}<0.05$ will be considered significant.

\section{Project status and timelines}

The recruitment of restaurants started in April 2019. Baseline survey was conducted between May and June 2019. One hundred and ninety-two restaurants from 12 counties completed the assessments, with data of 976 laboratory-tested dishes, 8145 recipes and 3840 customers. Theoretically, the follow-up survey will be conducted in the middle of 2020, after 12 months' intervention. Due to the pandemic of COVID-19 in early 2020, restaurants were temporarily closed in all the provinces of China. According to the feedback from the 12 counties, all of restaurants in our study were closed from late January to early April. We will evaluate influences of the COVID-19 pandemic on restaurants, especially on those in the intervention group, and decide on whether to postpone the follow-up assessment and other issues.

\section{Expected outcome and potential impact}

Unlike the standardised menus found in Western fast food chains, Chinese dishes vary considerably by areas, restaurants and chefs, even for dishes with the same name. Few studies existed on the sodium content of restaurant dishes in China. ${ }^{1425}$ The current study covered a wide range of restaurants in six provinces could provide the evidence on the sodium level and sources in Chinese restaurants, which will be very helpful to develop the specific intervention measures. The study aimed to explore a feasible, effective and sustainable approach to achieve salt reduction for Chinese restaurants. With an increasing proportion of people eating out, restaurant dishes contributed much more sodium than decades before and induced more health risks. Compared with salt reduction initiatives in individuals, communities or schools, those conducted in restaurants may face more challenges. With this study, we will identify barriers and facilitators for implementation, as well as solutions suitable to Chinese restaurants to reduce salt. Based on these findings, we will be able to draw important public health implications.

\section{Patient and public involvement}

Using information on the current situation of Chinese restaurants, inclusion criteria were determined before the recruitment. An open letter was disseminated via local media, introducing the RIS project and calling for participation publicly. Local CDC investigators, with support from the Administration for Market Regulation, made various mobilisation efforts to help restaurant owners understand the purpose of the project and the information to be collected in the investigation. With the consent of selected restaurants, we conducted assessment surveys and implemented the intervention. At the end of the study, we will disseminate the results to the restaurants and discuss with related stakeholders how to translate the research findings into practice and develop a public health strategy.

\section{ETHICS AND DISSEMINATION}

The study has been reviewed and approved by the Review Board of the National Institute for Nutrition and Health, China CDC (20180314), and Queen Mary Research Ethics Committee (QMERC2018/14).

According to the results of ethics review, written informed consent from restaurants and consumers were exempted from the RIS project. However, the investigators should fully inform the selected restaurants and consumers of the purpose and activities of the project, strive for understanding and cooperation and keep the information confidential. The restaurants and consumers will be free to discontinue their participation at any time without giving any reasons.

The findings of this research will be disseminated through conference presentations, peer-reviewed publications, press release and social media.

\section{Author affiliations}

${ }^{1}$ National Institute for Nutrition and Health, Chinese Center for Disease Control and Prevention, Beijing, China

${ }^{2}$ The George Institute for Global Health at Peking University Health Science Center, Beijing, China

${ }^{3}$ Wolfson Institute of Preventive Medicine, Queen Mary University of London, London, UK

${ }^{4}$ Heilongjiang Provincial Center for Disease Control and Prevention, Harbin, China ${ }^{5}$ Qinghai Provincial Center for Disease Control and Prevention, Xining, China ${ }^{6}$ Hebei Provincial Center for Disease Control and Prevention, Shijiazhuang, China ${ }^{7}$ Hunan Provincial Center for Disease Control and Prevention, Changsha, China ${ }^{8}$ Sichuan Provincial Center for Disease Control and Prevention, Chengdu, China

${ }^{9}$ Jiangxi Provincial Center for Disease Control and Prevention, Nanchang, China

Twitter Monique Tan @MoniqueTan91 and Rong Luo @0000-0003-2725-9939

Acknowledgements The authors would like to thank the restaurant managers, chefs and servicers, consumers, local administration for market regulation, experts of Sichuan Tourism University for their opinions on the development of the intervention program.

Contributors PZ and FJH conceived the project. WD, JZ, HW, YL, FJH and PZ participated in study design and implementation. WD, JZ, HW, YL, PZ, FJH, XZ, $Z X, Y G, L Y, X C$ and WY facilitates restaurant and public involvement and were responsible for setting up the study in each site. All authors contributed to the development of intervention and evaluation. WD wrote the first draft of the manuscript, and JZ, HW, YL, PZ, FJH, MT, GAM and RL revised the draft. All authors contributed to the refinement of the study protocol and approved the final manuscript.

Funding This work is supported by the National Institute for Health Research (NIHR, NIHR Global Health Research Unit Action on Salt China at Queen Mary University of London) using Official Development Assistance funding (16/136/77). 
Disclaimer The findings of this study will be disseminated through discussions or presentations at conferences, peer-reviewed publications and general media. The views expressed in this publication are those of the author(s) and not necessarily those of the NIHR or the Department of Health and Social Care.

Competing interests FJH is a member of the Consensus Action on Salt \& Health (CASH), a non-profit charitable organisation, and its international branch World Action on Salt \& Health (WASH). FJH does not receive any financial support from CASH or WASH. GAM is the chairman of Blood Pressure UK (BPUK), chairman of CASH and chairman of WASH and does not receive any financial support from any of these organisations. BPUK, CASH and WASH are non-profit charitable organisations. All other authors have no competing interests to declare.

Patient and public involvement Patients and/or the public were not involved in the design, or conduct, or reporting, or dissemination plans of this research.

Patient consent for publication Not required.

Provenance and peer review Not commissioned; externally peer reviewed.

Open access This is an open access article distributed in accordance with the Creative Commons Attribution Non Commercial (CC BY-NC 4.0) license, which permits others to distribute, remix, adapt, build upon this work non-commercially, and license their derivative works on different terms, provided the original work is properly cited, appropriate credit is given, any changes made indicated, and the use is non-commercial. See: http://creativecommons.org/licenses/by-nc/4.0/.

\section{ORCID iDs}

Wenwen Du http://orcid.org/0000-0002-4476-2985

Zhihua Xu http://orcid.org/0000-0002-0547-3355

Monique Tan http://orcid.org/0000-0003-4287-5553

\section{REFERENCES}

1 GBD 2017 Diet Collaborators. Health effects of dietary risks in 195 countries, 1990-2017: a systematic analysis for the global burden of disease study 2017. Lancet 2019;393:1958-72.

$2 \mathrm{He} \mathrm{Y,} \mathrm{Li} \mathrm{Y,} \mathrm{Yang} \mathrm{X,} \mathrm{et} \mathrm{al.} \mathrm{The} \mathrm{dietary} \mathrm{transition} \mathrm{and} \mathrm{its} \mathrm{association}$ with cardiometabolic mortality among Chinese adults, 1982-2012: a cross-sectional population-based study. Lancet Diabetes Endocrinol 2019;7:540-8.

3 Forouzanfar MH, Liu P, Roth GA, et al. Global burden of hypertension and systolic blood pressure of at least 110 to $115 \mathrm{MM} \mathrm{Hg}, 1990-$ 2015. JAMA 2017;317:165-82.

4 Bureau of National Health and Family Planning Commission Disease Prevention and Control. Report on Chinese residents' chronic diseases and nutrition. Beijing: People's Medical Publishing House, 2015.

5 Aburto NJ, Ziolkovska A, Hooper L, et al. Effect of lower sodium intake on health: systematic review and meta-analyses. BMJ 2013;346:f1326.

6 He FJ, Li J, Macgregor GA. Effect of longer term modest salt reduction on blood pressure: cochrane systematic review and metaanalysis of randomised trials. BMJ 2013;346:f1325.
7 Asaria P, Chisholm D, Mathers C, et al. Chronic disease prevention: health effects and financial costs of strategies to reduce salt intake and control tobacco use. Lancet 2007;370:2044-53.

8 Anderson CAM, Appel LJ, Okuda N, et al. Dietary sources of sodium in China, Japan, the United Kingdom, and the United States, women and men aged 40 to 59 years: the INTERMAP study. J Am Diet Assoc 2010;110:736-45.

9 He FJ, Wu Y, Feng X-X, et al. School based education programme to reduce salt intake in children and their families (School-EduSalt): cluster randomised controlled trial. BMJ 2015;350:h770.

10 Zhao F, Zhang P, Zhang L, et al. Consumption and sources of dietary salt in family members in Beijing. Nutrients 2015;7:2719-30.

11 Ahuja JKC, Wasswa-Kintu S, Haytowitz DB, et al. Sodium content of popular commercially processed and restaurant foods in the United States. Prev Med Rep 2015;2:962-7.

12 Wolfson JA, Moran AJ, Jarlenski MP, et al. Trends in sodium content of menu items in large chain restaurants in the U.S. Am J Prev Med 2018;54:28-36.

13 Prentice CA, Smith C, McLean RM. Sodium in commonly consumed fast foods in New Zealand: a public health opportunity. Public Health Nutr 2016;19:958-66.

14 Li X-qin, Wang Z, Men J-hua, et al. [Sodium content and energy of 47 commercial dishes in four restaurants in Beijing]. Zhonghua Yu Fang Yi Xue Za Zhi 2013;47:27-30.

15 An R. Fast-Food and full-service restaurant consumption and daily energy and nutrient intakes in US adults. Eur J Clin Nutr 2016;70:97-103.

16 Zang J, Luo B, Wang Y, et al. Eating out-of-home in adult residents in Shanghai and the nutritional differences among dining places. Nutrients 2018;10:nu10070951. doi:10.3390/nu10070951

17 Jia X, Liu J, Chen B, et al. Differences in nutrient and energy contents of commonly consumed dishes prepared in restaurants $\mathrm{V}$. at home in Hunan Province, China. Public Health Nutr 2018;21:1307-18.

18 He FJ, Zhang P, Li Y, et al. Action on salt China. Lancet 2018;392:7-9.

19 Zhang P, FJ H, Li Y, et al. Action on salt China (ASC): rationale and design (preprint). JMIR Research Protocols 2019;9.

20 He FJ, Zhang P, Luo R, et al. An Application-based programme to reinforce and maintain lower salt intake (AppSalt) in schoolchildren and their families in China. BMJ Open 2019;9:e027793.

21 Xu J, Tang B, Liu M, et al. A town level comprehensive intervention study to reduce salt intake in China: protocol for a cluster randomised controlled trial. BMJ Open 2020;10:e032976.

22 Ahn S-H, Kwon JS, Kim K, et al. Stages of behavioral change for reducing sodium intake in Korean consumers: comparison of characteristics based on social cognitive theory. Nutrients 2017;9:nu9080808. doi:10.3390/nu9080808

23 Ma GX, Shive S, Zhang Y, et al. Knowledge, perceptions, and behaviors related to salt use among Philadelphia Chinese take-out restaurant owners and chefs. Health Promot Pract 2014;15:638-45.

24 Zhang J, Sun L, Liu Y, et al. Mobile Device-Based electronic data capture system used in a clinical randomized controlled trial: advantages and challenges. J Med Internet Res 2017;19:e66.

25 Zhao N, Liang B, He P, et al. A survey on the sodium content of customers' orderings at three restaurants in Beijing. Zhonghua Liu Xing Bing Xue Za Zhi 2014;35:393-6. 